\title{
Universal relation between thermodynamic driving force and one-way fluxes in a nonequilibrium chemical reaction with complex mechanism
}

\author{
Yongli Peng, ${ }^{1, *}$ Hong Qian, ${ }^{2, \dagger}$ Daniel A. Beard, ${ }^{3, \ddagger}$ and Hao Ge $\odot^{4, \S}$ \\ ${ }^{1}$ School of Mathematical Science, Peking University, Beijing 100871, China \\ ${ }^{2}$ Department of Applied Mathematics, University of Washington, Seattle, Washington 98195, USA \\ ${ }^{3}$ Department of Molecular and Integrative Physiology, University of Michigan, Ann Arbor, Michigan 48105, USA \\ ${ }^{4}$ Beijing International Center for Mathematical Research (BICMR) and Biomedical Pioneering Innovation Center (BIOPIC), Peking \\ University, Beijing 100871, China
}

(Received 26 November 2019; revised 15 March 2020; accepted 16 June 2020; published 16 July 2020)

\begin{abstract}
In nonequilibrium chemical reaction systems, a fundamental relationship between unbalanced kinetic one-way fluxes and thermodynamic chemical driving forces is believed to exist. However, in fact, the formula for this fundamental relation, in general, and the very definition of kinetic one-way fluxes in nonlinear chemical reaction networks, are still missing. In terms of its stochastic kinetic representation, we formulate the one-way fluxes for a general chemical reaction far from equilibrium, with arbitrary complex mechanisms, multiple intermediates, and internal kinetic cycles. For each kinetic cycle, the logarithm of the ratio of the steady-state forward and backward one-way fluxes is equal to the free energy difference between the reactants and products along the cycle. This fundamental relation is further established for general chemical reaction networks with multiple input and output complexes. This work provides a concrete mathematical/physical basis for a long-hold belief in chemical kinetics. It gives an equivalent definition of the free energy difference in nonequilibrium chemical reaction networks and unifies the stochastic and macroscopic nonequilibrium chemical thermodynamics in a very broad setting.
\end{abstract}

DOI: 10.1103/PhysRevResearch.2.033089

\section{INTRODUCTION}

In the terminology of classical mechanics, chemical kinetics and thermodynamics correspond to kinematics and dynamics, respectively [1]. We show a universal relationship between kinetic fluxes and thermodynamic driving forces in a nonequilibrium chemical reaction network $(\mathrm{CRN})$, which are key concepts in the respective theories. More specifically, we prove

$$
\Delta G=-k_{B} T \ln \left(\frac{J^{+}}{J^{-}}\right),
$$

for a general, not necessarily elementary, chemical reaction, where $J^{+}$and $J^{-}$are forward and backward one-way fluxes and $\Delta G$ is the corresponding free energy difference. Equation (1) has been established for several special situations in the past [2-5]; the present work provides the most general theory to date.

\footnotetext{
*yonglipeng@pku.edu.cn

†ian@amath.washington.edu

*beardda@umich.edu

§haoge@pku.edu.cn
}

Published by the American Physical Society under the terms of the Creative Commons Attribution 4.0 International license. Further distribution of this work must maintain attribution to the author(s) and the published article's title, journal citation, and DOI.
Once Eq. (1) holds, the associated entropy production rate is immediately

$$
\text { epr }=-J \Delta G=k_{B} T\left(J^{+}-J^{-}\right) \ln \left(\frac{J^{+}}{J^{-}}\right) \geqslant 0,
$$

in which $J=J^{+}-J^{-}$is the net flux. The final equality in the above equation holds if and only if $J=\Delta G=0$, which implies the principle of detailed balance in thermochemical equilibrium [6]. Equation (1) is known to be closely related to the fluctuation theorem for entropy production [7-10].

For any stochastic reversible elementary reaction, one-way fluxes in both directions are well defined; they equal to the forward and backward reaction rates. For a complex CRN composed of many reversible elementary reactions, however, so far one-way flux in a nonequilibrium steady state (NESS) has been clearly defined only when all reactions are firstorder or pseudo-first-order, i.e., the kinetics is linear. In the nonlinear scenario, only net fluxes are clearly defined, which is exactly the difference between one-way fluxes and their reversed ones if they can be well defined. Therefore, how to generalize the concept of one-way flux together with Eq. (1) to nonlinear, nonelementary reactions is still an open problem [11], although people believe such a relation should be valid very generally.

The concept of one-way flux, introduced and extensively studied in [3] for linear CRN, relies on the notion of mean first passage time in stochastic processes. Hill's approach cannot be generalized to nonlinear chemical kinetics. This is the main 


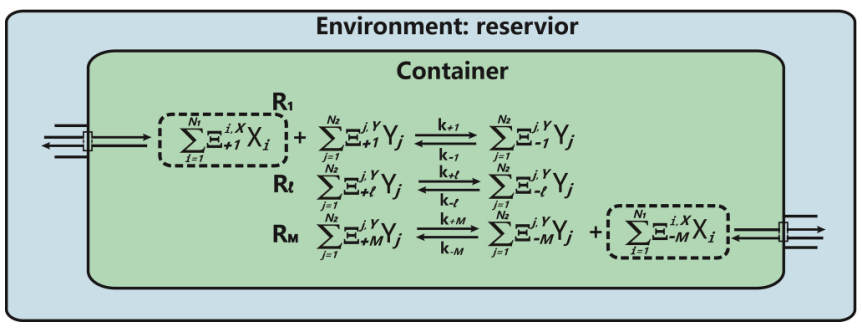

FIG. 1. Representation of a general CRN. $X$ 's are chemostated species and $Y$ 's are internal species, e.g., intermediates in the complete mechanism for transforming $X$ 's on the left to those on the right.

obstacle for introducing one-way flux into a general stochastic CRN, which had cast doubt on the universality of Eq. (1) [12].

In this article, we consider the general CRN in a NESS sustained by a chemostat [13]. The one-way flux of each reaction cycle is defined through the cycle fluxes in the counting space of the corresponding chemical-master-equation model [14] describing the stochastic kinetics of molecular numbers. Our theory is based on the general mathematical results on cycle fluxes of a Markov process [15,16]: We prove Eq. (1) for each reaction cycle by summing over all the corresponding stochastic cycles in the counting space and derive the entropy production rate in terms of the one-way cycle fluxes. Finally, we generalize all these results to the most general cases with the presence of multiple material reservoirs.

\section{CHEMICAL REACTION WITH COMPLEX MECHANISMS}

\section{A. Model}

A general CRN in a continuously stirred tank reactor consists of a set of species, $X_{1}, X_{2}, \ldots, X_{N_{1}}, Y_{1}, Y_{2}, \ldots, Y_{N_{2}}$, and a set of $M$ reactions between them, $R_{1}, R_{2}, \ldots, R_{M}$. Species are further classified into internal $Y$ 's and external $X$ 's. The concentrations of all the species $X$ 's are clamped at constant levels. All reactions are stochastic, elementary, and reversible, under isothermal and isobaric conditions with fixed volume $V$. A closed CRN has $N_{1}=0$.

The $M$ reactions, including those between the internal species and chemostated ones can be classified into the three groups:

$$
\begin{aligned}
& R_{1}: \sum_{j=1}^{N_{2}} \Xi_{+1}^{j, Y} Y_{j}+\sum_{i=1}^{N_{1}} \Xi_{+1}^{i, X} X_{i} \rightleftharpoons \sum_{j=1}^{N_{2}} \Xi_{-1}^{j, Y} Y_{j}, \\
& R_{\ell}: \sum_{j=1}^{N_{2}} \Xi_{+\ell}^{j, Y} Y_{j} \rightleftharpoons \sum_{j=1}^{N_{2}} \Xi_{-\ell}^{j, Y} Y_{j}, \\
& R_{M}: \sum_{j=1}^{N_{2}} \Xi_{+M}^{j, Y} Y_{j} \rightleftharpoons \sum_{i=1}^{N_{1}} \Xi_{-M}^{i, X} X_{i}+\sum_{j=1}^{N_{2}} \Xi_{-M}^{j, Y} Y_{j},
\end{aligned}
$$

where $\ell=2,3, \ldots, M-1$. Here only the reactions $R_{1}$ and $R_{M}$ exchange materials between $X$ and $Y$. Hence this reaction has a single input complex and a single output complex (Fig. 1). All the $Y$ 's species are intermediates in the mechanistic details of the overall reaction, transforming $\sum_{i=1}^{N_{1}} \Xi_{+1}^{i, X} X_{i}$ to $\sum_{i=1}^{N_{1}} \Xi_{-M}^{i, X} X_{i}$

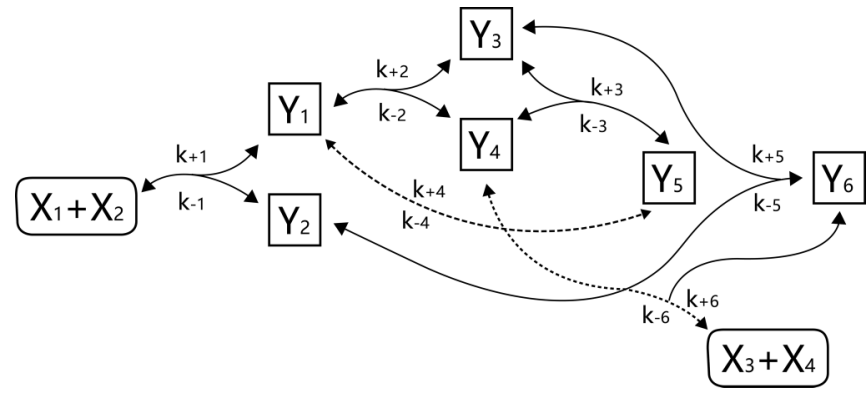

FIG. 2. An example with single input complex and single output complex, in which the Wegscheider cycle condition requires that $k_{2} k_{3} k_{-4}=k_{-2} k_{-3} k_{4}$.

Introducing the stoichiometric matrices $\boldsymbol{\Xi}^{X}$ and $\mathbf{\Xi}^{Y}$ for $X$ 's and $Y$ 's, respectively,

$$
\begin{aligned}
& \Xi^{X}=\left\{\Xi_{i \ell}^{X}=\Xi_{-\ell}^{i, X}-\Xi_{+\ell}^{i, X}\right\}_{N_{1} \times M}, \\
& \Xi^{Y}=\left\{\Xi_{j \ell}^{Y}=\Xi_{-\ell}^{j, Y}-\Xi_{+\ell}^{j, Y}\right\}_{N_{2} \times M} .
\end{aligned}
$$

The complete stoichiometric matrix, then, is $\boldsymbol{\Xi}=\left[\begin{array}{c}\boldsymbol{\Xi}^{X} \\ \boldsymbol{\Xi}^{Y}\end{array}\right]$. Note in this notion only reactions 1 and $M$ possess nonzero coefficients in $\boldsymbol{\Xi}^{X}$, for external species.

Figure 2 is an example with the overall reaction $X_{1}+$ $X_{2} \rightleftharpoons X_{3}+X_{4} . X_{1}, X_{2}, X_{3}$, and $X_{4}$ are the external species, with their concentrations kept as chemostated, and $Y_{1}$ to $Y_{6}$ are all intermediates.

We shall use $x_{i}(t)$ and $y_{j}(t)$ to denote the concentrations of $X_{i}$ and $Y_{j}$, respectively, at time $t$. Their macroscopic kinetics satisfies the rate equation:

$$
\frac{d y_{j}}{d t}=\sum_{\ell=1}^{M} \Xi_{j \ell}^{Y} J^{\ell}, \quad 1 \leqslant j \leqslant N_{2},
$$

where $J^{\ell}=J^{+\ell}-J^{-\ell}$ is the net flux of the $\ell$ th elementary reaction. $J^{ \pm \ell}$ are the macroscopic one-way fluxes satisfying the law of mass action [17-19], i.e.,

$$
J^{ \pm \ell}=k_{ \pm \ell} \prod_{j=1}^{N_{2}} y_{j} \Xi_{ \pm \ell}^{j, Y},
$$

except

$$
\begin{aligned}
J^{+1} & =k_{+1} \prod_{i=1}^{N_{1}} x_{i} \Xi_{+1}^{i, X} \prod_{j=1}^{N_{2}} y_{j} \Xi_{+1}^{j, Y}, \\
J^{-M} & =k_{-M} \prod_{i=1}^{N_{1}} x_{i} \Xi_{-M}^{i, X} \prod_{j=1}^{N_{2}} y_{j} \Xi_{-M}^{j, Y}
\end{aligned}
$$

$k_{ \pm \ell}$ are called the forward and backward chemical reaction rate constants for the $\ell$ th reaction.

Let $\mu_{j}^{Y}=\mu_{j}^{0, Y}+k_{B} T \log y_{j}$ be the chemical potential for $Y_{j}$, in which $\mu_{j}^{0, Y}$ is the standard chemical potential. Similar definitions also hold for $\mu_{i}^{X}$. Then for each single reaction $1 \leqslant$ $\ell \leqslant M$, one has [13] (also see Appendix A)

$$
k_{B} T \log \frac{J^{+\ell}}{J^{-\ell}}=\Delta G_{\ell}=G_{+\ell}-G_{-\ell},
$$


in which

$$
G_{+\ell}=\sum_{i=1}^{N_{1}} \Xi_{+\ell}^{i, X} \mu_{i}^{X}+\sum_{j=1}^{N_{2}} \Xi_{+\ell}^{j, Y} \mu_{j}^{Y}
$$

and

$$
G_{-\ell}=\sum_{i=1}^{N_{1}} \Xi_{-\ell}^{i, X} \mu_{i}^{X}+\sum_{j=1}^{N_{2}} \Xi_{-\ell}^{j, Y} \mu_{j}^{Y}
$$

are the total free energies of the reactants and products of the $\ell$ th reaction, respectively. Therefore, Eq. (2) is exactly Eq. (1) for any single macroscopic chemical reaction.

On the other hand, the mesoscopic setting focuses on molecular numbers instead of molar concentrations in the CRN, which are stochastic. We use $\mathbf{n}^{\mathbf{Y}}(t)=$ $\left[n_{1}^{Y}(t), n_{2}^{Y}(t), \ldots, n_{N_{2}}^{Y}(t)\right]$ to denote molecular numbers of the internal species at time $t$, which is a random vector. X's species still possess fixed molecular numbers, denoted by $\mathbf{n}^{\mathbf{x}}=\left(n_{1}^{x}, n_{2}^{x}, \ldots, n_{N_{1}}^{x}\right)$.

Next it is necessary to describe how $\mathbf{n}^{\mathbf{Y}}(t)$ evolves through time, which can be seen as a stochastic jumping process on a high-dimensional graph (called a counting space). Each vertex of the graph has an $N_{2}$-dimensional coordinate $\mathbf{n}^{\mathbf{y}}=$ $\left(n_{1}^{y}, n_{2}^{y}, \ldots, n_{N_{2}}^{y}\right)$ and if the occurrence of an elementary reaction in $\left\{R_{1}, \ldots, R_{M}\right\}$ can convert the state of the system from one vertex to another, then there is an edge connecting them in this graph.

The counting space is a scaffold for a Markov process with transition rates

$$
r_{+\ell}\left(\mathbf{n}^{\mathbf{y}} ; V\right) \triangleq \tilde{k}_{+\ell}(V) \prod_{j=1}^{N_{2}} n_{j}^{y}\left(n_{j}^{y}-1\right) \cdots\left(n_{j}^{y}-\Xi_{+\ell}^{j, Y}+1\right)
$$

for the forward reaction $R_{+\ell}$,

$$
r_{-\ell}\left(\mathbf{n}^{\mathbf{y}} ; V\right) \triangleq \tilde{k}_{-\ell}(V) \prod_{j=1}^{N_{2}} n_{j}^{y}\left(n_{j}^{y}-1\right) \cdots\left(n_{j}^{y}-\Xi_{-\ell}^{j, Y}+1\right)
$$

for the corresponding backward reaction $R_{-\ell}$, except two reactions involving $X$, i.e.,

$$
\begin{aligned}
r_{+1}\left(\mathbf{n}^{\mathbf{x}}, \mathbf{n}^{\mathbf{y}} ; V\right) \triangleq & \tilde{k}_{+1} \prod_{i=1}^{N_{1}} n_{i}^{x}\left(n_{i}^{x}-1\right) \cdots\left(n_{i}^{x}-\Xi_{+1}^{i, X}+1\right) \\
& \cdot \prod_{j=1}^{N_{2}} n_{j}^{y}\left(n_{j}^{y}-1\right) \cdots\left(n_{j}^{y}-\Xi_{+1}^{j, Y}+1\right)
\end{aligned}
$$

and

$$
\begin{aligned}
r_{-M}\left(\mathbf{n}^{\mathbf{x}}, \mathbf{n}^{\mathbf{y}} ; V\right) \triangleq & \tilde{k}_{-M} \prod_{i=1}^{N_{1}} n_{i}^{x}\left(n_{i}^{x}-1\right) \cdots\left(n_{i}^{x}-\Xi_{-M}^{i, X}+1\right) \\
& \cdot \prod_{j=1}^{N_{2}} n_{j}^{y}\left(n_{j}^{y}-1\right) \cdots\left(n_{j}^{y}-\Xi_{-M}^{j, Y}+1\right) .
\end{aligned}
$$

Here, $\tilde{k}_{ \pm \ell}$ are the mesoscopic rate constants in the stochastic model, and $k_{ \pm \ell}=\tilde{k}_{ \pm \ell} V^{n_{ \pm \ell}-1}$ where $n_{ \pm \ell}$ is the summation of stoichiometric numbers of reactants in the reaction $R_{ \pm \ell}$.
Then the chemical master equation (CME) describing the evolution of the probability $p_{V}\left(\mathbf{n}^{\mathbf{y}}, t\right)=\operatorname{Prob}\left(\mathbf{n}^{\mathbf{Y}}(t)=\mathbf{n}^{\mathbf{y}}\right)$ is

$$
\begin{aligned}
\frac{\partial p_{V}\left(\mathbf{n}^{\mathbf{y}}, t\right)}{\partial t}= & \sum_{\ell=2}^{M-1}\left\{r_{+\ell}\left(\mathbf{n}^{\mathbf{y}}-\Xi_{\ell}^{Y} ; V\right) p_{V}\left(\mathbf{n}^{\mathbf{y}}-\Xi_{\ell}^{Y}, t\right)\right. \\
& -\left[r_{+\ell}\left(\mathbf{n}^{\mathbf{y}} ; V\right)+r_{-\ell}\left(\mathbf{n}^{\mathbf{y}} ; V\right)\right] p_{V}\left(\mathbf{n}^{\mathbf{y}}, t\right) \\
& \left.+r_{-\ell}\left(\mathbf{n}^{\mathbf{y}}+\Xi_{\ell}^{Y} ; V\right) p_{V}\left(\mathbf{n}^{\mathbf{y}}+\Xi_{\ell}^{Y}, t\right)\right\} \\
& +\left\{r_{+1}\left(\mathbf{n}^{\mathbf{x}}, \mathbf{n}^{\mathbf{y}}-\Xi_{1}^{Y} ; V\right) p_{V}\left(\mathbf{n}^{\mathbf{y}}-\Xi_{1}^{Y}, t\right)\right. \\
& -\left[r_{+1}\left(\mathbf{n}^{\mathbf{x}}, \mathbf{n}^{\mathbf{y}} ; V\right)+r_{-1}\left(\mathbf{n}^{\mathbf{y}} ; V\right)\right] p_{V}\left(\mathbf{n}^{\mathbf{y}}, t\right) \\
& \left.+r_{-1}\left(\mathbf{n}^{\mathbf{y}}+\Xi_{1}^{Y} ; V\right) p_{V}\left(\mathbf{n}^{\mathbf{y}}+\Xi_{1}^{Y}, t\right)\right\} \\
& +\left\{r_{+M}\left(\mathbf{n}^{\mathbf{y}}-\Xi_{M}^{Y} ; V\right) p_{V}\left(\mathbf{n}^{\mathbf{y}}-\Xi_{M}^{Y}, t\right)\right. \\
& -\left[r_{+M}\left(\mathbf{n}^{\mathbf{y}} ; V\right)+r_{-M}\left(\mathbf{n}^{\mathbf{x}}, \mathbf{n}^{\mathbf{y}} ; V\right)\right] p_{V}\left(\mathbf{n}^{\mathbf{y}}, t\right) \\
& \left.+r_{-M}\left(\mathbf{n}^{\mathbf{x}}, \mathbf{n}^{\mathbf{y}}+\Xi_{M}^{Y} ; V\right) p_{V}\left(\mathbf{n}^{\mathbf{y}}+\Xi_{M}^{Y}, t\right)\right\},
\end{aligned}
$$

where $\boldsymbol{\Xi}_{\ell}^{Y}$ is just the $\ell$ th column of the matrix $\boldsymbol{\Xi}^{Y}$ with $1 \leqslant$ $\ell \leqslant M$.

\section{B. Kinetic cycle, net cycle flux, and one-way cycle fluxes}

A directed cycle in the counting space is a slice of path with the same origin and destination in which no other states overlap. It can be expressed in this way: $c=\left[\mathbf{y}_{1}, \mathbf{y}_{2}, \ldots, \mathbf{y}_{k}\right]$ with some integer $k$ where $\mathbf{y}_{1}, \mathbf{y}_{2}, \ldots, \mathbf{y}_{k}$ are successive states in the path which are different from each other. The next state of $\mathbf{y}_{k}$ in the path is exactly $\mathbf{y}_{1}$, forming a single closed loop. We identify $\left[\mathbf{y}_{1}, \mathbf{y}_{2}, \ldots, \mathbf{y}_{k}\right]$ and $\left[\mathbf{y}_{i}, \mathbf{y}_{i+1}, \ldots, \mathbf{y}_{i+k-1}\right]$ for any $i$ with indices modulo $k$, as the same directed cycle.

According to the theory of cycle representation and cycle fluxes for Markov process [16,20,21], one can trace along every sample path, and calculate the time-averaged number of times that a particular cycle $c$ formed by time $t$. Then letting the time $t$ go to infinity the limit defines the cycle flux $\omega_{c}$ for $c$. In the CME, the system is at equilibrium (time-reversible) if and only if $\omega_{c}=\omega_{c_{-}}$for every cycle $c[16,20]$, in which $c_{-}$ is the reversed cycle of $c$. Furthermore, the ratio

$$
\frac{\omega_{c}}{\omega_{c-}}=\frac{r_{\ell_{1}} r_{\ell_{2}} \cdots r_{\ell_{k}}}{r_{\ell_{1}^{-}} r_{\ell_{2}^{-}} \cdots r_{\ell_{k}^{-}}}
$$

where $\left\{\ell_{1}, \ell_{2}, \ldots, \ell_{k}\right\}$ represents the successive reactions $\left\{R_{\ell_{1}}, R_{\ell_{2}}, \ldots, R_{\ell_{k}}\right\}$ occurred in the cycle $c=\left[\mathbf{y}_{1}, \mathbf{y}_{2}, \ldots, \mathbf{y}_{k}\right]$, with $\ell_{i}^{-}$being the reversed reaction of the reaction $\ell_{i}$ and $r_{\ell}$ is the corresponding transition rates. The exact expression of cycle fluxes and the corresponding cycle decomposition of probability fluxes are given in Appendix B, cited from [16,20].

However, in many applications one is interested in the flux along reaction cycles rather than the cycle fluxes for any given cycle in the counting space. More specifically, such cycles, which we call reaction cycles, are right null vectors for the matrix $\Xi^{Y}$ [22]. We denote such a cycle as $\tilde{c}=\left(c_{1}, c_{2}, \ldots, c_{M}\right)$ in which $c_{\ell}$ is the net number of the occurrence for the reaction $R_{\ell}$. All the reaction cycles form a vector space.

Furthermore we can prove that the ratio $c_{1} / c_{M}$ is a positive invariant number depending on the topology of the network (see Appendix C), the simplest form of which is denoted as $\frac{c_{\text {in }}}{c_{\text {out }}}$. Therefore, we assume $c_{1}=n c_{\text {in }}$ and $c_{M}=n c_{\text {out }}$, where 
$n \in \mathbb{Z}$. The overall effect of such a reaction cycle is to make $\sum_{i} c_{\text {in }} \Xi_{+1}^{i, X} X_{i} \rightarrow \sum_{i} c_{\text {out }} \Xi_{-M}^{i, X} X_{i}$, which is just from the lefthand side of reaction $R_{1}$ to the right-hand side of reaction $R_{M}$ in Fig. 1, occurring $n$ times. Once $n=0$, then both $c_{1}=c_{M}=$ 0 , it is a closed cycle.

For each cycle $c$ in the counting space, the net numbers of occurrence of all the reactions in $c$ form a reaction cycle $\tilde{c}$. It follows naturally that the cycle flux of $\tilde{c}$ is defined as the sum of the cycle fluxes of all the counting cycles $c$ assorted to it. More formally we can define a map $\phi: \mathcal{C}_{\infty} \rightarrow \mathbb{R}^{M}$ with $\mathcal{C}_{\infty}$ being the set of all cycles in the counting space and $\phi(c)$ being the unique reaction cycle generated by $c$ as above. Then the cycle flux for $\tilde{c}$ can be interpreted as the averaged frequency $\tilde{c}$ occurs in the CME model. More precisely, the reaction cycle flux of $\tilde{c}$

$$
\omega_{\tilde{c}}=\sum_{\phi(c)=\tilde{c}} \omega_{c}
$$

It is also easy to verify that if a cycle $c$ is assigned to $\tilde{c}$, then its time reversal $c_{-}$will be assigned to $-\tilde{c}$. We thus just define the reversed reaction cycle $\tilde{c}_{-}$of $\tilde{c}$ as $-\tilde{c}$. Here we will show that for each cycle assigned to the same reaction cycle, the ratio of its flux and the flux of the corresponding reversed cycle is only dependent on how many times each reaction occurred in the cycle regardless of the order, being the same. To be more precise, we have

$$
\frac{\omega_{\tilde{c}}}{\omega_{\tilde{c}_{-}}}=\prod_{\ell=1}^{M} \frac{\tilde{k}_{+\ell}^{c_{\ell}}}{\tilde{k}_{-\ell}^{c_{\ell}}} \prod_{i=1}^{N_{1}} \frac{\left[n_{i}^{x}\left(n_{i}^{x}-1\right) \cdots\left(n_{i}^{x}-\Xi_{+1}^{i, X}+1\right)\right]^{c_{1}}}{\left[n_{i}^{x}\left(n_{i}^{x}-1\right) \cdots\left(n_{i}^{x}-\Xi_{-M}^{i, X}+1\right)\right]^{c_{M}}},
$$

which is equal to $\frac{\omega_{c}}{\omega_{c_{-}}}$for any $\phi(c)=\tilde{c}$.

In our model, the cycle in the counting space $c=$ $\left[\mathbf{y}_{1}, \mathbf{y}_{2}, \ldots, \mathbf{y}_{k}\right]$ for some integer $k$ and the transition rate denoted by $\left\{q_{\mathbf{n}^{\mathbf{y}} \cdot \mathbf{n}^{\mathbf{y}}}\right\}$ in the CME model is either

$$
\begin{aligned}
& q_{\mathbf{n}^{\mathbf{y}}\left(\mathbf{n}^{\mathbf{y}}+\Xi_{\ell}^{Y}\right)}=r_{+\ell}\left(\mathbf{n}^{\mathbf{y}} ; V\right),(2 \leqslant \ell \leqslant M), \\
& q_{\mathbf{n}^{\mathbf{y}}\left(\mathbf{n}^{\mathbf{y}}-\Xi_{\ell}^{Y}\right)}=r_{-\ell}\left(\mathbf{n}^{\mathbf{y}} ; V\right),(1 \leqslant \ell \leqslant M-1),
\end{aligned}
$$

or

$$
\begin{gathered}
q_{\mathbf{n}^{\mathbf{y}}\left(\mathbf{n}^{\mathbf{y}}+\Xi_{1}^{Y}\right)}=r_{+1}\left(\mathbf{n}^{\mathbf{x}}, \mathbf{n}^{\mathbf{y}} ; V\right), \\
q_{\mathbf{n}^{\mathbf{y}}\left(\mathbf{n}^{\mathbf{y}}-\Xi_{M}^{Y}\right)}=r_{-M}\left(\mathbf{n}^{\mathbf{x}}, \mathbf{n}^{\mathbf{y}} ; V\right) .
\end{gathered}
$$

Therefore if $2 \leqslant \ell \leqslant M-1$, and $y_{1}^{j}-\Xi_{+\ell}^{j, Y}=y_{2}^{j}-\Xi_{-\ell}^{j, Y}$ $\left(y_{1}^{j}, y_{2}^{j}\right.$ are the entries for $\left.\mathbf{y}_{1}, \mathbf{y}_{2}\right)$,

$$
\begin{aligned}
\frac{q_{\mathbf{y}_{1} \mathbf{y}_{2}}}{q_{\mathbf{y}_{2} \mathbf{y}_{1}}} & =\frac{\tilde{k}_{+\ell}}{\tilde{k}_{-\ell}} \cdot \prod_{j=1}^{N_{2}} \frac{y_{1}^{j}\left(y_{1}^{j}-1\right) \cdots\left(y_{1}^{j}-\Xi_{+\ell}^{j, Y}+1\right)}{y_{2}^{j}\left(y_{2}^{j}-1\right) \cdots\left(y_{2}^{j}-\Xi_{-\ell}^{j, Y}+1\right)} \\
& =\frac{\tilde{k}_{+\ell}}{\tilde{k}_{-\ell}} \cdot \prod_{j=1}^{N_{2}} \frac{\frac{\left(y_{1}^{j}\right) !}{\left(y_{1}^{j}-\Xi_{+\ell}^{j, Y}\right) !}}{\frac{\left(y_{2}^{j}\right) !}{\left(y_{2}^{j}-\Xi_{-\ell}^{j, Y}\right) !}} \\
& =\frac{\tilde{k}_{+\ell}}{\tilde{k}_{-\ell}} \cdot \prod_{j=1}^{N_{2}} \frac{\left(y_{1}^{j}\right) !}{\left(y_{2}^{j}\right) !} .
\end{aligned}
$$

$$
\begin{aligned}
& \text { If } \ell=1 \text {, and } y_{1}^{j}-\Xi_{+1}^{j, Y}=y_{2}^{j}-\Xi_{-1}^{j, Y} \text {, } \\
& \frac{q_{\mathbf{y}_{1} \mathbf{y}_{2}}}{q_{\mathbf{y}_{2} \mathbf{y}_{1}}}=\frac{\tilde{k}_{+1} \prod_{i} n_{i}^{x}\left(n_{i}^{x}-1\right) \cdots\left(n_{i}^{x}-\Xi_{+1}^{i, X}+1\right)}{\tilde{k}_{-1}} \\
& \times \prod_{j=1}^{N_{2}} \frac{y_{1}^{j}\left(y_{1}^{j}-1\right) \cdots\left(y_{1}^{j}-\Xi_{+1}^{j, Y}+1\right)}{y_{2}^{j}\left(y_{2}^{j}-1\right) \cdots\left(y_{2}^{j}-\Xi_{-1}^{j, Y}+1\right)} \\
& =\frac{\tilde{k}_{+1} \prod_{i} n_{i}^{x}\left(n_{i}^{x}-1\right) \cdots\left(n_{i}^{x}-\Xi_{+1}^{i, X}+1\right)}{\tilde{k}_{-1}} \prod_{j=1}^{N_{2}} \frac{\left(y_{1}^{j}\right) !}{\left(y_{2}^{j}\right) !} .
\end{aligned}
$$

If $\ell=M$, and $y_{1}^{j}-\Xi_{+M}^{j, Y}=y_{2}^{j}-\Xi_{-M}^{j, Y}$,

$$
\frac{q_{\mathbf{y}_{1} \mathbf{y}_{2}}}{q_{\mathbf{y}_{2} \mathbf{y}_{1}}}=\frac{\tilde{k}_{+M}}{\tilde{k}_{-M} \prod_{i} n_{i}^{x} \cdots\left(n_{i}^{x}-\Xi_{-M}^{i, X}+1\right)} \prod_{j=1}^{N_{2}} \frac{\left(y_{1}^{j}\right) !}{\left(y_{2}^{j}\right) !} .
$$

The above formulas remain the same for all the transitions $\mathbf{y}_{1} \rightarrow \mathbf{y}_{2}, \ldots, \mathbf{y}_{k} \rightarrow \mathbf{y}_{1}$. Then for the reaction cycle $\tilde{c}=\phi(c)$, we apply the formula in Appendix B and obtain

$$
\begin{aligned}
\frac{\omega_{c}}{\omega_{c-}} & =\frac{q_{\mathbf{y}_{1} \mathbf{y}_{2}}}{q_{\mathbf{y}_{2} \mathbf{y}_{1}}} \frac{q_{\mathbf{y}_{2} \mathbf{y}_{3}}}{q_{\mathbf{y}_{3} \mathbf{y}_{2}}} \cdots \frac{q_{\mathbf{y}_{k} \mathbf{y}_{1}}}{q_{\mathbf{y}_{1} \mathbf{y}_{k}}} \\
& =\prod_{\ell=2}^{M-1} \frac{\tilde{k}_{+\ell}^{c_{\ell}}}{\tilde{k}_{-\ell}^{c_{\ell}}} \frac{\tilde{k}_{+M}^{c_{M}}}{\tilde{k}_{-1}^{c_{1}}} \frac{\left[\tilde{k}_{+1} \prod_{i} n_{i}^{x} \cdots\left(n_{i}^{x}-\Xi_{+1}^{i, X}+1\right)\right]^{c_{1}}}{\left[\tilde{k}_{-M} \prod_{i} n_{i}^{x} \cdots\left(n_{i}^{x}-\Xi_{-M}^{i, X}+1\right)\right]^{c_{M}}} \\
& =\prod_{\ell=1}^{M} \frac{\tilde{k}_{+\ell}^{c_{\ell}}}{\tilde{k}_{-\ell}^{c_{\ell}}} \prod_{i=1}^{N_{1}} \frac{\left[n_{i}^{x}\left(n_{i}^{x}-1\right) \cdots\left(n_{i}^{x}-\Xi_{+1}^{i, X}+1\right)\right]^{c_{1}}}{\left[n_{i}^{x}\left(n_{i}^{x}-1\right) \cdots\left(n_{i}^{x}-\Xi_{-M}^{i, X}+1\right)\right]^{c_{M}}} .
\end{aligned}
$$

One can see from this formula that the ratio is invariant for any cycle satisfying $\phi(c)=\tilde{c}=\left(c_{1}, c_{2}, \ldots, c_{M}\right)$. Then we have $\omega_{c} / \omega_{c_{-}}=\omega_{\tilde{c}} / \omega_{\tilde{c}_{-}}$.

Once $c_{1}=c_{M}=0$, i.e., a closed cycle, the ratio is 1 according to the Wegscheidier's condition, i.e., $\prod_{\ell=2}^{M-1} \frac{\tilde{k}_{+\ell}^{c_{\ell}}}{\tilde{\kappa}_{-\ell}^{c_{\ell}}}=1$, indicating no driving force available for the cycle. In a closed system with only closed cycles, where there are no input and output reactions $R_{1}$ and $R_{M}$ present, $\frac{\omega_{\tilde{c}}}{\omega_{\tilde{c}}}=1$ for each reaction cycle, which is the hallmark of equilibrium state.

Equation (4) is the first main result of this article, which will lead to the generalization of Eqs. (1) and (2) for each reaction cycle. Typically, the external species $X$ is controlled macroscopically in the unit of molar concentration $x_{i}=$ $V^{-1} n_{i}^{x}$. Notice $k_{ \pm \ell}=\tilde{k}_{ \pm \ell} V^{n_{ \pm \ell}-1}$, and replace the molecular number by the molar concentration, i.e., $x_{i}=n_{i}^{x} / V$, then

$$
\frac{\omega_{c}}{\omega_{c_{-}}}=\prod_{\ell=1}^{M}\left(\frac{k_{+\ell} V^{n_{-\ell}}}{k_{-\ell} V^{n_{+\ell}}}\right)^{c_{\ell}} \cdot \prod_{i=1}^{N_{1}} \frac{\left[x_{i} V \cdots\left(x_{i} V-\Xi_{+1}^{i, X}+1\right)\right]^{c_{1}}}{\left[x_{i} V \cdots\left(x_{i} V-\Xi_{-M}^{i, X}+1\right)\right]^{c_{M}}} .
$$

Define the macroscopic cycle currents for the reaction cycle $\tilde{c}$ as $\mathcal{J}_{\tilde{c}}=\lim _{V \rightarrow \infty} \frac{\omega_{\tilde{c}}}{V}$. Let $V \rightarrow \infty,\left(x_{i} V-m\right) \approx x_{i} V$ for any fixed $m \in \mathbb{N}$, then the total power of $\mathrm{V}$ on the righthand side of the above formula is easy to be shown as zero, which is a direct result of $\boldsymbol{\Xi}^{Y} \cdot \tilde{c}=0$. Thus

$$
\frac{\mathcal{J}_{\tilde{c}}}{\mathcal{J}_{\tilde{c}_{-}}}=\left[\prod_{\ell=1}^{M} \frac{k_{+\ell}^{c_{\ell}}}{k_{-\ell}^{c_{\ell}}}\right] \cdot \prod_{i=1}^{N_{1}}\left(x_{i}\right)^{\Xi_{+1}^{i, X} c_{1}-\Xi_{-M}^{i, X} c_{M}} .
$$


Since $k_{B} T \log \prod_{\ell=1}^{M} \frac{k_{++}^{c_{\ell}}}{k_{+\ell}^{c_{\ell}}}$ is equal to $n \Delta G_{1 \rightarrow M}^{0}$, in which $\Delta G_{1 \rightarrow M}^{0}=\sum_{i}\left(c_{\text {in }} \Xi_{+1}^{i, X}-c_{\text {out }} \Xi_{-M}^{i, X}\right) \mu_{i}^{0, X}$ is the intrinsic free energy difference of the overall reaction $\sum_{i} c_{\text {in }} \Xi_{+1}^{i, X} X_{i} \rightarrow$ $\sum_{i} c_{\text {out }} \Xi_{-M}^{i, X} X_{i}$ (using the relation between reaction rates and chemical potentials in Appendix A), then

$$
\begin{aligned}
k_{B} T & \log \frac{\mathcal{J}_{\tilde{c}}}{\mathcal{J}_{\tilde{c}_{-}}} \\
= & n \Delta G_{1 \rightarrow M}^{0}+n \sum_{i=1}^{N_{1}}\left(c_{\text {in }} \Xi_{+1}^{i, X}-c_{\text {out }} \Xi_{-M}^{i, X}\right) k_{B} T \log x_{i} \\
& =n \sum_{i}\left(c_{\text {in }} \Xi_{+1}^{i, X}-c_{\text {out }} \Xi_{-M}^{i, X}\right) \mu_{i}^{X}=n \Delta G_{1 \rightarrow M},
\end{aligned}
$$

which is exactly the free energy difference of the overall reaction.

We can also obtain the corresponding cycle decomposition of the macroscopic fluxes $J^{\ell}$ in terms of the reaction cycles (more detailed decomposition is derived in Appendixes D and E):

$$
J^{\ell}=\sum_{\tilde{c} \in \phi\left(\mathcal{C}_{\infty}\right)} c_{\ell} \cdot \mathcal{J}_{\tilde{c}}
$$

This is exactly the relation that used for the definition of reaction-cycle currents in [13,22]. Let $\left\{\tilde{c}^{\gamma}, \tilde{c}^{\alpha}\right\}_{1 \leqslant \gamma \leqslant A, 1 \leqslant \alpha \leqslant B}$ be the basis spanning the right null space of $\Xi^{Y}$, where $\tilde{c}^{\gamma}$ are closed and $\tilde{c}^{\alpha}$ are nonclosed. If $\tilde{c}=\sum_{\gamma} s_{\gamma}^{\tilde{c}} \tilde{c}^{\gamma}+\sum_{\alpha} s_{\alpha}^{\tilde{c}} \tilde{c}^{\alpha}$, then according to Eq. (7) [here $j^{*}=\left(J^{\ell}\right)_{1 \leqslant \ell \leqslant M}$ is the steady-state macroscopic flux],

$$
j^{*}=\sum_{\tilde{c}} \mathcal{J}_{\tilde{c}} \tilde{c}=\sum_{\gamma}\left(\sum_{\tilde{c}} \mathcal{J}_{\tilde{c}} s_{\gamma}^{\tilde{c}}\right) \tilde{c}_{\gamma}+\sum_{\alpha}\left(\sum_{\tilde{c}} \mathcal{J}_{\tilde{c}} s_{\alpha}^{\tilde{c}}\right) \tilde{c}_{\alpha} .
$$

Therefore, $\mathcal{J}_{\gamma}=\sum_{\tilde{c}} \mathcal{J}_{\tilde{c}} s_{\gamma}^{\tilde{c}}$ and $\mathcal{J}_{\alpha}=\sum_{\tilde{c}} \mathcal{J}_{\tilde{c}} s_{\alpha}^{\tilde{c}}$ are just the coordinates of $j^{*}$ under this basis, which are exactly the cycle currents of $\tilde{c}^{\gamma}$ and $\tilde{c}^{\alpha}$ defined in [22].

However, such a definition is not unique, dependent on the choice of basis. Here in our definition, all the reactioncycle fluxes are exactly their averaged frequency of occurrence per unit time at steady state, and we are able to distinguish the cycle fluxes of the original cycles and their reversed ones, making the definition of one-way fluxes plausible.

Furthermore, once a reaction cycle is completed, a certain number $(n)$ of $\sum_{i} c_{\text {in }} \Xi_{+1}^{i, X} X_{i}$ is transformed into $\sum_{i} c_{\text {out }} \Xi_{-M}^{i, X} X_{i}$, which is the overall effect of a reaction cycle. Many reaction cycles share the same overall effect. So we can define the oneway flux upon the overall effect, namely the value of $n$ in this single input/output scenario, i.e.,

$$
J_{n}=\sum_{c_{1}=n c_{\mathrm{in}}} \omega_{\tilde{c}}, \forall n \in \mathbb{Z} .
$$

We will show the function $F(\tilde{c})=\log \frac{\omega_{\tilde{c}}}{\omega_{\tilde{c}_{-}}}$is a linear function only depends on $n$, the overall effect.
According to Eq. (4), for the reaction cycle $\tilde{c}=$ $\left(c_{1}, c_{2}, \ldots, c_{M}\right), F(\tilde{c})=\sum_{j=1}^{M} c_{j} F_{j}$, where

$$
\begin{aligned}
& F_{1}=\log \frac{\tilde{k}_{+1}}{\tilde{k}_{-1}}+\sum_{i=1}^{N_{1}} \log \left[n_{i}^{x}\left(n_{i}^{x}-1\right) \cdots\left(n_{i}^{x}-\Xi_{+1}^{i, X}+1\right)\right], \\
& F_{M}=\log \frac{\tilde{k}_{+M}}{\tilde{k}_{-M}}-\sum_{i=1}^{N_{1}} \log \left[n_{i}^{x}\left(n_{i}^{x}-1\right) \cdots\left(n_{i}^{x}-\Xi_{-M}^{i, X}+1\right)\right], \\
& F_{\ell}=\log \frac{\tilde{k}_{+\ell}}{\tilde{k}_{-\ell}} .
\end{aligned}
$$

for $\ell=2,3, \ldots, M-1$. So $F(\tilde{c})$ is explicitly a linear function of $\tilde{c}$.

Next, we shall show $F(\tilde{c})$ is only dependent on the overall effect $n$ of $\tilde{c}$. If there is another $\tilde{c}^{\prime}=\left(n c_{\text {in }}, c_{2}^{\prime}, \ldots, c_{M-1}^{\prime}, n c_{\text {out }}\right)$ with the same overall effect but $F(\tilde{c}) \neq F\left(\tilde{c}^{\prime}\right)$, then $\tilde{e}=\tilde{c}-$ $\tilde{c}^{\prime}$ is still a reaction cycle and $F(\tilde{e})=F(\tilde{c})-F\left(\tilde{c}^{\prime}\right) \neq 0$. The reactions $R_{1}, R_{M}$ do not occur in $\tilde{e}$, so it is a closed cycle only involving $\left(Y_{1}, \ldots, Y_{N_{2}}\right)$. But according to the Wegscheider's condition [23], $F(\tilde{e})=0$ and this is a contradiction.

Since the term involving $n_{i}^{x}$ in $F(\tilde{c})$ [see Eq. (4)] is already a linear function only depends on $n$, so does $\log \prod_{\ell=1}^{M} \frac{\tilde{k}_{+\ell}^{c_{\ell}}}{\tilde{k}_{-\ell}^{c_{\ell}}}$ and denoted it as $n \cdot \kappa$. Then

$$
\begin{aligned}
\frac{J_{n}}{J_{-n}} & =\frac{\sum_{c_{1}=n c_{\text {in }}} \omega_{\tilde{c}}}{\sum_{c_{1}=-n c_{\text {in }}} \omega_{\tilde{c}}} \\
& =e^{n \kappa} \prod_{i=1}^{N_{1}} \frac{\left[n_{i}^{x}\left(n_{i}^{x}-1\right) \cdots\left(n_{i}^{x}-\Xi_{+1}^{i, X}+1\right)\right]^{n c_{\text {in }}}}{\left[n_{i}^{x}\left(n_{i}^{x}-1\right) \cdots\left(n_{i}^{x}-\Xi_{-M}^{i, X}+1\right)\right]^{n c_{\text {out }}}} .
\end{aligned}
$$

Taking $V \rightarrow \infty$, we also obtain

$$
k_{B} T \log \frac{\mathcal{J}_{n}}{\mathcal{J}_{-n}}=n \Delta G_{1 \rightarrow M},
$$

where we use the similar notation $\mathcal{J}_{n}=\lim _{V \rightarrow \infty} \frac{J_{n}}{V}$ for the macroscopic one-way flux dependent only on the overall effect.

\section{Steady-state entropy production rate}

The steady-state mesoscopic entropy production rate (mesoEPR) in the counting space can be expressed by all the cycle fluxes [16]

$$
\operatorname{mesoEPR}=\frac{1}{2} k_{B} T \sum_{c}\left(\omega_{c}-\omega_{c_{-}}\right) \log \frac{\omega_{c}}{\omega_{c_{-}}} .
$$

In terms of reaction cycles, another decomposition of mesoEPR is straightforwardly obtained:

$$
\operatorname{mesoEPR}=\frac{1}{2} k_{B} T \sum_{\tilde{c}}\left(\omega_{\tilde{c}}-\omega_{\tilde{c}_{-}}\right) \log \frac{\omega_{\tilde{c}}}{\omega_{\tilde{c}_{-}}}=\sum_{\tilde{c}} \omega_{\tilde{c}} \mathcal{A}_{\tilde{c}}^{\text {meso }},
$$

in which $\mathcal{A}_{\tilde{c}}^{\text {meso }}=k_{B} T \log \frac{\omega_{\tilde{\tau}}}{\omega_{\overline{c_{-}}}}$. 
Similarly, the mesoEPR can also be decomposed by the overall effect of reaction cycles:

$$
\begin{aligned}
\operatorname{mesoEPR} & =\frac{1}{2} k_{B} T \sum_{n=-\infty}^{\infty} \sum_{c_{1}=n c_{\text {in }}}\left(\omega_{\tilde{c}}-\omega_{\tilde{c}_{-}}\right) \log \frac{\omega_{\tilde{c}}}{\omega_{\tilde{c}_{-}}} \\
& =\frac{1}{2} k_{B} T \sum_{n=-\infty}^{\infty}\left(J_{n}-J_{-n}\right) \log \frac{J_{n}}{J_{-n}} \\
& =\frac{1}{2} k_{B} T \sum_{n=-\infty}^{\infty} n\left(J_{n}-J_{-n}\right) \log \frac{J_{1}}{J_{-1}} \\
& =k_{B} T J_{T} \log \frac{J_{1}}{J_{-1}}
\end{aligned}
$$

noticing $\frac{J_{n}}{J_{-n}}=\left[\frac{J_{1}}{J_{-1}}\right]^{n}$ and $J_{T}=\sum_{n=-\infty}^{\infty} n J_{n}$ is the total net flux of $X$.

Towards the macroscopic scale as the volume goes to infinity, it has been recently proved that [24] the limit of mesoEPR $/ V$ is exactly the steady-state macroscopic entropy production rate defined as

$$
\operatorname{macroEPR}=\frac{1}{2} k_{B} T \sum_{\ell=-M, \ell \neq 0}^{M}\left(J^{+\ell}-J^{-\ell}\right) \log \frac{J^{+\ell}}{J^{-\ell}} .
$$

Since

$$
\frac{\mathcal{J}_{\tilde{c}}}{\mathcal{J}_{\tilde{c}_{-}}}=\prod_{\ell=1}^{M}\left(\frac{J^{+\ell}}{J^{-\ell}}\right)^{c_{\ell}},
$$

and combined with the cycle decomposition of the macroscopic fluxes [Eq. (7)], we have the cycle decomposition of macroscopic entropy production rate

$$
\begin{aligned}
\operatorname{macroEPR} & =k_{B} T \sum_{\ell=1}^{M} J^{\ell} \log \frac{J^{+\ell}}{J^{-\ell}} \\
& =k_{B} T \sum_{\ell=1}^{M}\left[\sum_{\tilde{c} \in \phi\left(\mathcal{C}_{\infty}\right)} \mathcal{J}_{\tilde{c}} c_{\ell}\right] \log \frac{J^{+\ell}}{J^{-\ell}} \\
& =k_{B} T \sum_{\tilde{c} \in \phi\left(\mathcal{C}_{\infty}\right)} \mathcal{J}_{\tilde{c}}\left[\sum_{\ell=1}^{M} c_{\ell} \log \frac{J^{+\ell}}{J^{-\ell}}\right] \\
& =\sum_{\tilde{c}} \mathcal{J}_{\tilde{c}} \mathcal{A}_{\tilde{c}}^{\text {macro }}=\mathcal{J}_{T} \Delta G_{1 \rightarrow M},
\end{aligned}
$$

in which

$$
\mathcal{A}_{\tilde{c}}^{\text {macro }}=k_{B} T \log \frac{\mathcal{J}_{\tilde{c}}}{\mathcal{J}_{\tilde{c}_{-}}}=k_{B} T \sum_{\ell=1}^{M} c_{\ell} \ln \frac{J^{+\ell}}{J^{-\ell}}
$$

is just the change of Gibbs free energy along $\tilde{c}$, called cycle affinity [13,22], and $\mathcal{J}_{T}=\sum_{n=-\infty}^{\infty} n \mathcal{J}_{n}$ is the total net macroscopic flux. Equation (10) can also be derived by the means of taking $V \rightarrow \infty$ for mesoEPR/V in Eq. (8) and Eq. (9), noticing the limit of $\mathcal{A}_{\tilde{c}}^{\text {meso }}$ is exactly $\mathcal{A}_{\tilde{c}}^{\text {macro }}$.

Furthermore, the expression of $\mathcal{A}_{\tilde{c}}^{\text {macro }}$ shows that it is linear w.r.t. $\tilde{c}$. So if $\tilde{c}=\sum_{\gamma} s_{\gamma}^{\tilde{c}} \tilde{c}^{\gamma}+\sum_{\alpha} s_{\alpha}^{\tilde{c}} \tilde{c}^{\alpha}$, we have $\mathcal{A}_{\tilde{c}}^{\text {macro }}=$ $\sum_{\gamma} s_{\gamma}^{\tilde{c}} \mathcal{A}_{\gamma}+\sum_{\alpha} s_{\alpha}^{\tilde{c}} \mathcal{A}_{\alpha}$, where $\mathcal{A}_{\gamma}, \mathcal{A}_{\alpha}$ are the corresponding cycle affinity for the basis $\left\{\tilde{c}^{\gamma}, \tilde{c}^{\alpha}\right\}_{1 \leqslant \gamma \leqslant A, 1 \leqslant \alpha \leqslant B}$, in which
$\mathcal{A}_{\gamma}=0$ for closed cycles. Thus

$$
\begin{aligned}
\text { macroEPR } & =\sum_{\tilde{c}} \mathcal{J}_{\tilde{c}} \mathcal{A}_{\tilde{c}}^{\text {macro }} \\
& =\sum_{\alpha}\left[\sum_{\tilde{c}} \mathcal{J}_{\tilde{c}} s_{\alpha}^{\tilde{c}}\right] \mathcal{A}_{\alpha}=\sum_{\alpha} \mathcal{J}_{\alpha} \mathcal{A}_{\alpha},
\end{aligned}
$$

which is just what obtained in [22].

\section{GENERALIZATION TO GENERAL CRN WITH MULTIPLE INPUT AND OUTPUT COMPLEXES}

In the general scenario with multiple input and output complexes, we assume the reactions are

$$
\begin{aligned}
& R_{\varrho}^{b}: \sum_{j} \Xi_{+\varrho, b}^{j, Y} Y_{j}+\sum_{i} \Xi_{+\varrho, b}^{i, X} X_{i} \\
& \rightleftharpoons \sum_{j} \Xi_{-\varrho, b}^{j, Y} Y_{j}+\sum_{i} \Xi_{-\varrho, b}^{i, X} X_{i}, \\
& R_{\rho}^{i}: \sum_{j} \Xi_{+\rho, i}^{j, Y} Y_{j} \rightleftharpoons \sum_{j} \Xi_{-\rho, i}^{j, Y} Y_{j},
\end{aligned}
$$

where $1 \leqslant \varrho \leqslant B$ and $1 \leqslant \rho \leqslant I$. The species are still $\left(X_{1}, X_{2}, \ldots, X_{N_{1}}, Y_{1}, Y_{2}, \ldots, Y_{N_{2}}\right)$ with $X$ 's "outside" and $Y$ 's "inside." Then the stoichiometric matrix shall be

$$
\Xi=\left[\begin{array}{cc}
\Xi_{b}^{X} & 0 \\
\Xi_{b}^{Y} & \Xi_{i}^{Y}
\end{array}\right],
$$

in which

$$
\Xi_{b}^{X}=\left\{\Xi_{\varrho, b}^{i, X}=-\Xi_{+\varrho, b}^{i, X}+\Xi_{-\varrho, b}^{i, X}\right\}_{N_{1} \times B}
$$

and

$$
\Xi_{b}^{Y}=\left\{\Xi_{\varrho, b}^{j, Y}=-\Xi_{+\varrho, b}^{j, Y}+\Xi_{-\varrho, b}^{j, Y}\right\}_{N_{2} \times B}
$$

indicate the stoichiometric matrix for $X$ and $Y$ separately with "boundary" reactions, namely reactions between the chemostatted species and the internal ones. $\Xi_{i}^{Y}=$ $\left\{\Xi_{\rho, i}^{j, Y}=-\Xi_{+\rho, i}^{j, Y}+\Xi_{-\rho, i}^{j, Y}\right\}_{N_{2} \times I}$ is the matrix with "internal" reactions only involving $Y$ 's.

As for the transition rates, we have

$$
r_{+\rho, i}\left(\mathbf{n}^{\mathbf{y}} ; V\right) \triangleq \tilde{k}_{+\rho, i}(V) \prod_{j=1}^{N_{2}} n_{j}^{y}\left(n_{j}^{y}-1\right) \cdots\left(n_{j}^{y}-\Xi_{+\rho, i}^{j, Y}+1\right)
$$

and

$$
\begin{aligned}
r_{+\varrho, b}\left(\mathbf{n}^{\mathbf{x}}, \mathbf{n}^{\mathbf{y}} ; V\right) \triangleq & \tilde{k}_{+\varrho, b}(V) \prod_{i} n_{i}^{x}\left(n_{i}^{x}-1\right) \\
& \cdots\left(n_{i}^{x}-\Xi_{+\varrho, b}^{i, X}+1\right) \cdot \prod_{j} n_{j}^{y}\left(n_{j}^{y}-1\right) \\
& \cdots\left(n_{j}^{y}-\Xi_{+\varrho, b}^{j, Y}+1\right)
\end{aligned}
$$

for forward internal and boundary reactions. The rates for backward reactions can be defined in the same way.

Then the chemical master equation can be formulated as below (here $\Xi_{\varrho, b}^{Y}$ and $\Xi_{\rho, i}^{Y}$ are just the $\varrho$ th columns of the matrix $\Xi_{b}^{Y}$ and $\Xi_{i}^{Y}$ respectively with $1 \leqslant \varrho \leqslant B$ and $1 \leqslant \rho \leqslant$ 
I):

$$
\begin{aligned}
\frac{\partial p_{V}\left(\mathbf{n}^{\mathbf{y}}, t\right)}{\partial t}= & \sum_{\rho=1}^{I}\left\{r_{+\rho, i}\left(\mathbf{n}^{\mathbf{y}}-\Xi_{\rho, i}^{Y} ; V\right) p_{V}\left(\mathbf{n}^{\mathbf{y}}-\Xi_{\rho, i}^{Y}, t\right)\right. \\
& -\left[r_{+\rho, i}\left(\mathbf{n}^{\mathbf{y}} ; V\right)+r_{-\rho, i}\left(\mathbf{n}^{\mathbf{y}} ; V\right)\right] p_{V}\left(\mathbf{n}^{\mathbf{y}}, t\right) \\
& \left.+r_{-\rho, i}\left(\mathbf{n}^{\mathbf{y}}+\Xi_{\rho, i}^{Y} ; V\right) p_{V}\left(\mathbf{n}^{\mathbf{y}}+\Xi_{\rho, i}^{Y}, t\right)\right\} \\
& +\sum_{\varrho=1}^{B}\left\{r_{+\varrho, b}\left(\mathbf{n}^{\mathbf{x}}, \mathbf{n}^{\mathbf{y}}-\Xi_{\varrho, b}^{Y} ; V\right) p_{V}\left(\mathbf{n}^{\mathbf{y}}-\Xi_{\varrho, b}^{Y}, t\right)\right. \\
& -\left[r_{+\varrho, b}\left(\mathbf{n}^{\mathbf{x}}, \mathbf{n}^{\mathbf{y}} ; V\right)+r_{-\varrho, b}\left(\mathbf{n}^{\mathbf{x}}, \mathbf{n}^{\mathbf{y}} ; V\right)\right] \\
& \times p_{V}\left(\mathbf{n}^{\mathbf{y}}, t\right)+r_{-\varrho, b}\left(\mathbf{n}^{\mathbf{x}}, \mathbf{n}^{\mathbf{y}}+\Xi_{\varrho, b}^{Y} ; V\right) \\
& \left.\times p_{V}\left(\mathbf{n}^{\mathbf{y}}+\Xi_{\varrho, b}^{Y}, t\right)\right\} .
\end{aligned}
$$

The cycles $c$ in the counting space and associated cycle fluxes $\omega_{c}$ are also defined in the CME model, and Eq. (3) still holds here, i.e.,

$$
\frac{\omega_{c}}{\omega_{c_{-}}}=\frac{r_{\ell_{1}} r_{\ell_{2}} \cdots r_{\ell_{k}}}{r_{\ell_{1}^{-}} r_{\ell_{2}^{-}} \cdots r_{\ell_{k}^{-}}}
$$

where $\left\{\ell_{1}, \ell_{2}, \ldots, \ell_{k}\right\}$ represents the successive reactions occurred in the cycle $c$, with $\ell_{i}^{-}$being the reversed reaction of the reaction $\ell_{i}$ and $r_{\ell}$ is the corresponding transition rate.

The reaction cycle $\tilde{c}$ is still defined as the right null vector of $\Xi^{Y}=\left[\Xi_{b}^{Y}, \Xi_{i}^{Y}\right]$, denoted as $\tilde{c}=$ $\left(b_{1}, b_{2}, \ldots, b_{B}, i_{1}, i_{2}, \ldots, i_{I}\right)=\left(\vec{b}^{T}, \vec{i}^{T}\right)$. Using the same map $\phi: \mathcal{C}_{\infty} \rightarrow \mathbb{R}^{I+B}$ we can define the reaction cycle flux:

$$
\omega_{\tilde{c}}=\sum_{\phi(c)=\tilde{c}} \omega_{c}
$$

With similar calculations to the previous sections, we can thus derive

$$
\begin{aligned}
\frac{\omega_{\tilde{c}}}{\omega_{\tilde{c}_{-}}}= & \prod_{\rho=1}^{I} \frac{\tilde{k}_{+\rho, i}^{i_{\rho}}}{\tilde{k}_{-\rho, i}^{i_{\rho}}} \prod_{\varrho=1}^{B} \frac{\tilde{k}_{+\varrho, b}^{b_{\varrho}}}{\tilde{k}_{-\varrho, b}^{b_{\varrho}}} \\
& \prod_{\varrho=1}^{B}\left[\prod_{i=1}^{N_{1}} \frac{n_{i}^{x}\left(n_{i}^{x}-1\right) \cdots\left(n_{i}^{x}-\Xi_{+\varrho, b}^{i, X}+1\right)}{n_{i}^{x}\left(n_{i}^{x}-1\right) \cdots\left(n_{i}^{x}-\Xi_{-\varrho, b}^{i, X}+1\right)}\right]^{b_{\varrho}} .
\end{aligned}
$$

When the molecular numbers $n_{i}^{x}$ of $\mathrm{X}$ are very large, we use the molar concentration $x_{i}=V^{-1} n_{i}^{x}$ and the macroscopic cycle current $\mathcal{J}_{\tilde{c}}$ instead, then the following result follows:

$$
\frac{\mathcal{J}_{\tilde{c}}}{\mathcal{J}_{\tilde{c}_{-}}}=\left[\prod_{\rho=1}^{I} \frac{k_{+\rho, i}^{i_{\rho}}}{k_{-\rho, i}^{i_{\rho}}} \prod_{\varrho=1}^{B} \frac{k_{+\varrho, b}^{b_{\varrho}}}{k_{-\varrho, b}^{b_{\ell}}}\right] \prod_{\varrho=1}^{B}\left[\prod_{i=1}^{N_{1}}\left(x_{i}\right)^{-\Xi_{\varrho, b}^{i, X}}\right]^{b_{\varrho}},
$$

in which these $k$ 's without ${ }^{\sim}$ are the reaction constants in the corresponding macroscopic kinetics.

Then we can similarly prove the free energy difference $\Delta G$ is, in fact, the logarithm of the above ratio multiplied by $k_{B} T$ in the macroscopic scale.
Similar to the relations in Appendix A, here we have for any $1 \leqslant \varrho \leqslant B$,

$$
\begin{aligned}
k_{B} T \log \frac{k_{+\varrho, b}}{k_{-\varrho, b}}= & \sum_{j=1}^{N_{2}}\left(\Xi_{+\varrho, b}^{j, Y}-\Xi_{-\varrho, b}^{j, Y}\right) \mu_{j}^{0, Y} \\
& +\sum_{i=1}^{N_{1}}\left(\Xi_{+\varrho, b}^{i, X}-\Xi_{-\varrho, b}^{i, X}\right) \mu_{i}^{0, X}
\end{aligned}
$$

and for any $1 \leqslant \rho \leqslant I$,

$$
k_{B} T \log \frac{k_{+\rho, i}}{k_{-\rho, i}}=\sum_{j=1}^{N_{2}}\left(\Xi_{+\rho, i}^{j, Y}-\Xi_{-\rho, i}^{j, Y}\right) \mu_{j}^{0, Y},
$$

followed by

$$
\begin{aligned}
& k_{B} T \log \left[\prod_{\rho=1}^{I} \frac{k_{+\rho, i}^{i_{\rho}}}{k_{-\rho, i}^{i_{\rho}}} \prod_{\varrho=1}^{B} \frac{k_{+\varrho, b}^{b_{e}}}{k_{-\varrho, b}^{b_{e}}}\right] \\
&=-\left(\mu^{0, X}, \mu^{0, Y}\right)\left[\begin{array}{cc}
\Xi_{b}^{X} & 0 \\
\Xi_{b}^{Y} & \Xi_{i}^{Y}
\end{array}\right] \tilde{c}^{T} \\
&=-\left(\mu^{0, X}, \mu^{0, Y}\right)\left[\begin{array}{l}
\vec{l} \\
0
\end{array}\right]=-\vec{l} \cdot \mu^{0, X},
\end{aligned}
$$

and

$$
k_{B} T \log \left(\prod_{\varrho=1}^{B}\left[\prod_{i=1}^{N_{1}}\left(x_{i}\right)^{-\Xi_{e, b}^{i, X}}\right]^{b_{\ell}}\right)=-\vec{p}^{T} \cdot \Xi_{b}^{X} \cdot \vec{b}=-\vec{p} \cdot \vec{l},
$$

where $\quad \vec{p}=\left(k_{B} T \log x_{i}\right)_{1 \leqslant i \leqslant N_{1}}, \quad$ and $\quad \vec{l}=\left[\Xi_{b}^{X}, 0\right] \times \tilde{c}^{T}=$ $\left[\Xi_{b}^{X}, 0\right][\vec{b}]=\Xi_{b}^{X} \vec{b}$ is the overall effect of $\tilde{c}$.

Hence if we let $V$ goes to infinity, combined with Eq. (13), the limit of the mesoscopic cycle affinity $\mathcal{A}_{\tilde{c}}^{\text {meso }}=k_{B} T \log \frac{\omega_{\bar{c}}}{\omega_{\bar{c}}}$ is

$$
\mathcal{A}_{\tilde{c}}^{\text {macro }}=k_{B} T \log \frac{\mathcal{J}_{\tilde{c}}}{\mathcal{J}_{\tilde{c}_{-}}}=-\vec{l} \cdot \mu_{X}=\Delta G_{\tilde{c}},
$$

in which $\Delta G_{\tilde{c}}$ is the free energy difference along the reaction cycle $\tilde{c}$, which is only dependent on the overall effect $\vec{l}$. It is the force-flux relation under the most general setting to date.

Furthermore, we can show that the ratio $\frac{\omega_{\bar{c}}}{\omega_{\bar{c}_{-}}}$at the mesoscopic scale has already been only dependent on the overall effect $\vec{l}$ (see Appendix F). Therefore for any vector $\vec{l}$, we can also group all the reaction cycles with the same overall effect together, defining the mesoscopic one-way flux $J_{\vec{l}}$ for the overall effect $\vec{l}$ :

$$
J_{\vec{l}}=\sum_{\left[\Xi_{b}^{X}, 0\right] \times \tilde{c}=\vec{l}} \omega_{\tilde{c}} .
$$

Thus the macroscopic one-way flux for the overall effect

$$
\mathcal{J}_{\vec{l}}=\lim _{V \rightarrow \infty} \frac{J_{\vec{l}}}{V}=\sum_{\left[\Xi_{b}^{X}, 0\right] \times \tilde{c}=\vec{l}} \mathcal{J}_{\tilde{c}} .
$$

Therefore, the ratio

$$
k_{B} T \log \frac{\mathcal{J}_{\vec{l}}}{\mathcal{J}_{-\vec{l}}}=-\vec{l} \cdot \mu_{X} \triangleq \Delta G_{\vec{l}},
$$


where $\Delta G_{\vec{l}}$ encodes the free energy difference of the overall reaction $\sum_{i} l_{i}^{-} X_{i} \rightarrow \sum_{i} l_{i}^{+} X_{i}$ where $\vec{l}=\left(l_{1}, l_{2}, \cdots, l_{N_{1}}\right)^{T}$, $l_{i}^{-}=-\min \left\{0, l_{i}\right\}$ and $l_{i}^{+}=\max \left\{0, l_{i}\right\}$ are $\vec{l}$ 's negative and positive parts.

Finally, the steady-state mesoscopic entropy production rate (mesoEPR) in the counting space keeps several levels of cycle decomposition, i.e.,

$$
\begin{aligned}
\operatorname{mesoEPR} & =\frac{1}{2} k_{B} T \sum_{c}\left(\omega_{c}-\omega_{c_{-}}\right) \log \frac{\omega_{c}}{\omega_{c_{-}}} \\
& =\frac{1}{2} k_{B} T \sum_{\tilde{c}}\left(\omega_{\tilde{c}}-\omega_{\tilde{c}_{-}}\right) \log \frac{\omega_{\tilde{c}}}{\omega_{\tilde{c}_{-}}}=\sum_{\tilde{c}} \omega_{\tilde{c}} \mathcal{A}_{\tilde{c}}^{\text {meso }} \\
& =\frac{1}{2} k_{B} T \sum_{\vec{l}}\left(J_{\vec{l}}-J_{-\vec{l}}\right) \log \frac{J_{\vec{l}}}{J_{-\vec{l}}} .
\end{aligned}
$$

Taking $V \rightarrow \infty$ for mesoEPR/ $V$, the steady-state macroscopic entropy production rate

$$
\begin{aligned}
\operatorname{macroEPR} & =\frac{1}{2} k_{B} T \sum_{\ell}\left(J^{+\ell}-J^{-\ell}\right) \log \frac{J^{+\ell}}{J^{-\ell}} \\
& =\sum_{\tilde{c}} \mathcal{J}_{\tilde{c}} \mathcal{A}_{\tilde{c}}^{\text {macro }},
\end{aligned}
$$

where $J^{ \pm \ell}$ is the classic macroscopic one-way flux for the "boundary" reaction $R_{ \pm \ell}^{b}$ when $\ell \leqslant B$ and the flux for the "internal" reaction $R_{ \pm(\ell-B)}^{i}$ when $\ell>B$.

According to Eq. (16), we can further obtain

$$
\operatorname{macroEPR}=\sum_{\vec{l}} \mathcal{J}_{\vec{l}} \Delta G_{\vec{l}}=-j_{X} \cdot \mu_{X},
$$

where $j_{X}=\left(\sum_{\vec{l}} \mathcal{J}_{\vec{l}} \cdot \vec{l}\right)$ denotes the overall steady-state flux of external species $X$. In flux balance analysis (FBA) $[25,26]$ and energy balance analysis (EBA) [27], $-j_{X}$ are just the injection fluxes maintaining the external species $X$ 's concentrations. For example, in the metabolic network, they can be the uptake and secretion rates of the relevant metabolites.

\section{CONCLUSION AND DISCUSSION}

Using the theory of cycle representation for Markov processes, mesoscopic, and macroscopic one-way cycle fluxes in a nonlinear complex CRN are rigorously defined. Cycle decomposition are carried out at three different levels, including mesoscopic counting space, emergent cycle space, and macroscopic CRN; the force-flux relation is demonstrated for each reaction cycle. These provided a consistent computation for entropy production. The universal relation between one-way fluxes and thermodynamic driving force proven in the present work underpins a consistency between kinetics and thermodynamics. It introduces new perspectives into and builds bridges between theories of CRNs at different scales. Our theory finally turns people's previous beliefs of such a general force-flux relation into a solid theory, from definitions to proofs.

\section{ACKNOWLEDGMENT}

H.G. was supported by NSFC (Grants No. 11971037 and 11622101).

\section{APPENDIX A: FORCE-FLUX RELATION FOR A MACROSCOPIC SINGLE CHEMICAL REACTION}

In this section, we attempt to derive the force-flux relation for a single macroscopic chemical reaction

$$
k_{B} T \log \frac{J^{+\ell}}{J^{-\ell}}=\Delta G_{\ell},
$$

for each reaction $R_{\ell}$, where $J^{ \pm \ell}$ are macroscopic one-way fluxes and $\Delta G_{\ell}$ is the corresponding free energy difference.

The chemical potential of $Y_{j}$ is defined as $\mu_{j}^{Y}=\mu_{j}^{0, Y}+$ $k_{B} T \log y_{j}$, and similar definition holds for $X_{i}$. Therefore the total free energies of the reactants and products of the $\ell$ th reaction are $G_{ \pm \ell}=\sum_{i=1}^{N_{1}} \Xi_{ \pm \ell}^{i, X} \mu_{i}^{X}+\sum_{j=1}^{N_{2}} \Xi_{ \pm \ell}^{j, Y} \mu_{j}^{Y}$.

Hence

$$
\begin{aligned}
\Delta G_{\ell}= & G_{+\ell}-G_{-\ell} \\
= & -\sum_{i=1}^{N_{1}} \Xi_{\ell}^{i, X} \mu_{i}^{0, X}-\sum_{j=1}^{N_{2}} \Xi_{\ell}^{j, Y} \mu_{j}^{0, Y} \\
& -k_{B} T \sum_{i=1}^{N_{1}} \Xi_{\ell}^{i, X} \log x_{i}-k_{B} T \sum_{j=1}^{N_{2}} \Xi_{\ell}^{j, Y} \log y_{j},
\end{aligned}
$$

in which $\Xi_{\ell}^{i, X}=\Xi_{-\ell}^{i, X}-\Xi_{+\ell}^{i, X}, \Xi_{\ell}^{j, Y}=\Xi_{-\ell}^{j, Y}-\Xi_{+\ell}^{j, Y}$

At thermodynamic equilibrium, we have $\Delta G_{\ell}=0$, followed by

$$
\begin{aligned}
& \sum_{i=1}^{N_{1}} \Xi_{\ell}^{i, X} \mu_{i}^{0, X}+\sum_{j=1}^{N_{2}} \Xi_{\ell}^{j, Y} \mu_{j}^{0, Y} \\
& \quad=-k_{B} T \sum_{i=1}^{N_{1}} \Xi_{\ell}^{i, X} \log x_{i}^{\mathrm{eq}}-k_{B} T \sum_{j=1}^{N_{2}} \Xi_{\ell}^{j, Y} \log y_{j}^{\mathrm{eq}} .
\end{aligned}
$$
i.e.,

At thermodynamic equilibrium, we also have $J^{+\ell}=J^{-\ell}$,

$$
k_{+\ell} \prod_{i=1}^{N_{1}}\left(x_{i}^{\mathrm{eq}}\right)^{\Xi_{+\ell}^{i, x}} \prod_{j=1}^{N_{2}}\left(y_{j}^{\mathrm{eq}}\right)^{\Xi_{+\ell}^{j, Y}}=k_{-\ell} \prod_{i=1}^{N_{1}}\left(x_{i}^{\mathrm{eq}}\right)^{\Xi_{-\ell}^{i, x}} \prod_{j=1}^{N_{2}}\left(y_{j}^{\mathrm{eq}}\right)^{\Xi_{-\ell}^{j, Y}} .
$$

Thus we obtain

$$
\sum_{i=1}^{N_{1}} \Xi_{\ell}^{i, X} \mu_{i}^{0, X}+\sum_{j=1}^{N_{2}} \Xi_{\ell}^{j, Y} \mu_{j}^{0, Y}=-k_{B} T \log \frac{k_{+\ell}}{k_{-\ell}} .
$$

Finally we have the force-flux relation

$$
\begin{aligned}
\Delta G_{\ell} & =k_{B} T \log \frac{k_{+\ell} \prod_{i=1}^{N_{1}}\left(x_{i}\right)^{\Xi_{+\ell}^{i, x}} \prod_{j=1}^{N_{2}}\left(y_{j}\right)^{\Xi_{+\ell}^{j, Y}}}{k_{-\ell} \prod_{i=1}^{N_{1}}\left(x_{i}\right)^{\Xi_{-\ell}^{i, x}} \prod_{j=1}^{N_{2}}\left(y_{j}\right)^{\Xi_{-\ell}^{j, Y}}} \\
& =k_{B} T \log \frac{J^{+\ell}}{J^{-\ell}} .
\end{aligned}
$$




\section{APPENDIX B: EXPRESSIONS OF CYCLE FLUXES AND CYCLE DECOMPOSITION IN ANY MARKOV JUMPING PROCESSES}

In this section we will list the exact expression of cycle fluxes in the context of a general continuous-time Markov jumping process with countable states, including the chemical-master-equation model on the counting space. The detailed derivation can be referred to $[16,20]$.

Suppose $\xi=\left\{\xi_{t}\right\}_{t \in \mathrm{R}}$ is a Markov jumping process with a countable state space $S=\{1,2, \ldots$,$\} and a transition density$ matrix $Q=\left\{q_{y \tilde{y}}\right\}$ satisfying

$$
\sum_{\tilde{y} \in S, \tilde{y} \neq y} q_{y \tilde{y}}=-q_{y y} \triangleq q_{y}<\infty,
$$

then along a single trajectory, one can calculate the frequency of each given cycle's occurrence up to time $t$. As the time goes to infinity, the limit gives the cycle flux, and the expression of cycle flux is

$\omega_{c}$

$$
\begin{aligned}
= & (-1)^{s-1}\left[\sum_{y \in S} \pi_{y} q_{y}\right] \frac{q_{y_{1} y_{2}} q_{y_{2} y_{3}} \ldots q_{y_{s} y_{1}}}{q_{y_{1}} q_{y_{2}} \ldots q_{y_{s}}} \tilde{\pi}_{y_{1}} g\left(y_{2}, y_{2} \mid\left\{y_{1}\right\}\right) \\
& \cdot g\left(y_{3}, y_{3} \mid\left\{y_{1}, y_{2}\right\}\right) \ldots g\left(y_{s}, y_{s} \mid\left\{y_{1}, y_{2}, \ldots, y_{s-1}\right\}\right)
\end{aligned}
$$

for the given cycle $c=\left[y_{1}, y_{2}, \ldots, y_{s}\right]$, where $\left(\pi_{y}\right)_{y \in S}$ is the invariant distribution of $\xi,\left(\tilde{\pi}_{y}\right)_{y \in S}$ is the invariant distribution of the imbedded Markov chain $\tilde{\xi}$, and $g\left(j, k \mid\left\{y_{1}, \ldots, y_{l}\right\}\right)$ denotes the taboo Green's function of the imbedded Markov chain $\tilde{\xi}$ :

$$
\begin{aligned}
& g\left(y, \tilde{y} \mid\left\{y_{1}, y_{2}, \ldots, y_{l}\right\}\right) \\
& \quad=\sum_{n=0}^{\infty} \mathbf{P}\left(\tilde{\xi}_{n}=\tilde{y}, \tilde{\xi}_{m} \notin\left\{y_{1}, y_{2}, \ldots, y_{l}\right\}, \forall 1 \leqslant m<n \mid \tilde{\xi}_{0}=y\right) .
\end{aligned}
$$

The imbedded Markov chain is given by $\tilde{\xi}_{n}=\xi_{T_{n}}$, in which $T_{n}$ denotes the $n$th jumping time of the process. It follows that $\tilde{\xi}=\left\{\tilde{\xi}_{n}\right\}_{n \geqslant 0}$ is a discrete time Markov chain with the transition matrix $P=\left(\tilde{p}_{y \tilde{y}}\right)_{y, \tilde{y} \in S}$ where

$$
\tilde{p}_{y \tilde{y}}= \begin{cases}\frac{q_{y \tilde{y}}}{q_{y}}, & y \neq \tilde{y}, \\ 0, & y=\tilde{y} .\end{cases}
$$

Moreover, if we assume both $\xi$ and $\tilde{\xi}$ have unique invariant distributions $\left(\pi_{y}\right)_{y \in S}$ and $\left(\tilde{\pi}_{y}\right)_{y \in S}$, then

$$
\tilde{\pi}_{y}=\frac{\pi_{y} q_{y}}{\sum_{\tilde{y} \in S} \pi_{\tilde{y}} q_{\tilde{y}}} .
$$

In particular, in the case when $S$ is a finite space,

$$
\omega_{c}=(-1)^{s-1} q_{y_{1} y_{2}} q_{y_{2} y_{3}} \ldots q_{y_{s} y_{1}} \frac{\left|Q\left(\left\{y_{1}, y_{2}, \ldots, y_{s}\right\}^{c}\right)\right|}{\sum_{j \in S}\left|Q\left(\{j\}^{c}\right)\right|},
$$

where we use $|Q(H)|$ to denote the determinant of $Q$ with rows and columns indexed by $H$.

Furthermore, one can have the cycle decomposition of onestep probability flux $[16,20]$ :

$$
\pi_{y} q_{y \tilde{y}}=\sum_{c \in \mathcal{C}_{\infty}} w_{c} J_{c}(y, \tilde{y}), \quad \forall y, \tilde{y} \in S .
$$

Here $\mathcal{C}_{\infty}$ is the class of all cycles occurring during the process, and

It is followed by

$$
J_{c}(y, \tilde{y})=\left\{\begin{array}{l}
1, \text { if }(y, \tilde{y}) \text { is an edge of } c ; \\
0, \text { otherwise. }
\end{array}\right.
$$

$$
\pi_{y} q_{y \tilde{y}}-\pi_{\tilde{y}} q_{\tilde{y} y}=\sum_{c \in \mathcal{C}_{\infty}}\left(w_{c}-w_{c_{-}}\right) J_{c}(y, \tilde{y}),
$$

where $c_{-}$denotes the reversed cycle of $c$

\section{APPENDIX C: $c_{1} / c_{M}$ SHOULD BE A CONSTANT IN THE SINGLE INPUT/OUTPUT SCENARIO}

We show here the vector-like expression of any reaction cycle $\tilde{c}=\left(c_{1}, c_{2}, \ldots, c_{M}\right)$ shall maintain $c_{1} / c_{M}$ as a constant

Assume we have two such reaction cycles $\tilde{c}_{1}=$ $\left(c_{1}^{(1)}, c_{2}^{(1)}, \ldots, c_{M}^{(1)}\right)$ and $\tilde{c}_{2}=\left(c_{1}^{(2)}, c_{2}^{(2)}, \ldots, c_{M}^{(2)}\right)$.

If $c_{M}^{(2)} c_{1}^{(1)}>c_{M}^{(1)} c_{1}^{(2)}$, then define $\tilde{c}_{0}=c_{1}^{(1)} \tilde{c}_{2}-c_{1}^{(2)} \tilde{c}_{1}=$ $\left(0, c_{2}^{(2)} c_{1}^{(1)}-c_{2}^{(1)} c_{1}^{(2)}, \ldots, c_{M}^{(2)} c_{1}^{(1)}-c_{M}^{(1)} c_{1}^{(2)}\right)$, which is also a reaction cycle and no reaction $R_{1}$ happened in it. So the overall effect once such a reaction cycle takes place, will leave all $\left(Y_{1}, \ldots, Y_{N_{2}}\right)$ unchanged, but produce certain $X$ 's through the reaction $R_{-M}$. But this will never be true since each reaction should be mass conserved.

More mathematically, let the vector $m \in \mathbb{R}^{\left(N_{1}+N_{2}\right) \times 1}$ be the mass of each species including $X$ 's and $Y$ 's and the conservation of mass gives $m^{T} \Xi=0$. We further assume that the total mass of $Y$ 's will increase during the reaction $R_{1}$ and decrease during the reaction $R_{M}$, i.e., $I=m^{T} \Xi_{1}^{Y}>0$ and $O=m^{T} \Xi_{M}^{Y}<0$, where $\Xi_{1}^{Y}, \Xi_{M}^{Y}$ are the 1 st and $M$ th columns of $\boldsymbol{\Xi}^{Y}$. In this case the submatrix $\tilde{\boldsymbol{\Xi}}^{Y}=\left\{\boldsymbol{\Xi}_{j \ell}^{Y}\right\}_{\left(1 \leqslant j \leqslant N_{2} ; 2 \leqslant l \leqslant M-1\right)}$ is closed and $m^{T} \tilde{\Xi}^{Y}=0, m^{T} \boldsymbol{\Xi}^{Y}=(I, 0, O)$.

Let $\tilde{e}_{0}=\left(0, c_{2}^{(2)} c_{1}^{(1)}-c_{2}^{(1)} c_{1}^{(2)}, \ldots, c_{M-1}^{(2)} c_{1}^{(1)}-c_{M-1}^{(1)} c_{1}^{(2)}, 0\right)$, then since $\Xi^{Y} \tilde{c}_{0}^{T}=0$, we have

$$
\boldsymbol{\Xi}^{Y} \tilde{e}_{0}^{T}=\left(c_{M}^{(2)} c_{1}^{(1)}-c_{M}^{(1)} c_{1}^{(2)}\right)\left(-\Xi_{M}^{Y}\right) .
$$

On the one hand, $m^{T} \boldsymbol{\Xi}^{Y} \tilde{e}_{0}^{T}=(I, 0, O) \cdot \tilde{e}_{0}=0$. On the other hand, $m^{T} \boldsymbol{\Xi}^{Y} \tilde{e}_{0}^{T}=(-O) \times\left(c_{M}^{(2)} c_{1}^{(1)}-c_{M}^{(1)} c_{1}^{(2)}\right)>0$ by (C1) and $O<0$. This is a contradiction and so $c_{M}^{(2)} c_{1}^{(1)} \leqslant$ $c_{M}^{(1)} c_{1}^{(2)}$.

Similarly $c_{M}^{(2)} c_{1}^{(1)} \geqslant c_{M}^{(1)} c_{1}^{(2)}$. So $c_{M}^{(2)} c_{1}^{(1)}=c_{M}^{(1)} c_{1}^{(2)}$.

This shall be satisfied for any pair of two reaction cycles $\tilde{c}_{1}$ and $\tilde{c}_{2}$, so if $c_{1}^{(1)}=0$ then $c_{M}^{(1)}=0$ and it is a closed cycle. If neither of them is zero, then the ratio $c_{1} / c_{M}$ must be maintained as a constant for any nonclosed reaction cycle.

We can further prove $c_{1} / c_{M}$ is positive whenever $c_{1} \neq 0$. If we assume $c_{1}<0$ and $c_{M}>0$, then we can consider the vector $\tilde{e}_{1}=\left(0, c_{2}, \ldots, c_{M-1}, 0\right)$ and we have

$$
\Xi^{Y} \tilde{e}_{1}^{T}=\left(-c_{1} \Xi_{1}^{Y}-c_{M} \Xi_{M}^{Y}\right) \text {. }
$$

By the conservation law we have $m^{T} \boldsymbol{\Xi}^{Y} \tilde{e}_{1}^{T}=0$. By (C2) we have $m^{T} \boldsymbol{\Xi}^{Y} \tilde{e}_{1}^{T}=-c_{1} I-c_{M} O>0$, which leads to contradiction. The case with $c_{1}>0$ and $c_{M}<0$ is similar. So eventually $c_{1} / c_{M}$ should be positive.

Thus we can assume $c_{1}=n c_{\text {in }}$ and $c_{M}=n c_{\text {out }}$ hereafter, where $n \in \mathbb{Z}, c_{\text {in }}$ and $c_{\text {out }}$ are the smallest positive integers maintaining the constant $c_{1} / c_{M}$. This indicates the overall effect of a reaction cycle is just to make $\sum_{i} c_{\text {in }} \Xi_{+1}^{i, X} X_{i} \rightarrow$ $\sum_{i} c_{\text {out }} \Xi_{-M}^{i, X} X_{i}$ happen $n$ times. 


\section{APPENDIX D: CYCLE DECOMPOSITION OF PROBABILITY FLUX IN TERMS OF REACTION CYCLES ON THE COUNTING SPACE OF CME}

Cycle decomposition of probability flux [Eqs. (B1) and (B2)] can be generalized in terms of reaction cycles. Here we should consider a more detailed definition of reaction cycles, called "directed reaction cycles," considering the two directions of each reaction separately. Therefore the corresponding stoichiometric matrices for $X$ and $Y$ are $\Xi_{d}^{X}=$ $\left\{\Xi_{\ell}^{i, X},-\Xi_{\ell}^{i, X}\right\}_{N_{1} \times 2 M}$ and $\Xi_{d}^{Y}=\left\{\Xi_{\ell}^{j, Y},-\Xi_{\ell}^{j, Y}\right\}_{N_{2} \times 2 M}$, and the total directed stoichiometric matrix $\Xi_{d}=\left[\begin{array}{l}\Xi_{d}^{X} \\ \Xi_{d}^{Y}\end{array}\right]$, is a $\left(N_{1}+\right.$ $\left.N_{2}\right) \times 2 M$ matrix with the $2 M$ columns ordered by the reactions $\left\{R_{+1}, R_{+2}, \ldots, R_{+M}, R_{-1}, R_{-2}, \ldots, R_{-M}\right\}$.

The directed reaction cycle is a $2 M$-dimensional vector expressed as $\left\{\tilde{c}_{d}=\left(c_{1}^{+}, c_{2}^{+}, \ldots, c_{M}^{+}, c_{1}^{-}, c_{2}^{-}, \ldots, c_{M}^{-}\right)\right\}$, which is a right null vector of the matrix $\Xi_{d}^{Y}$. Then for each cycle $c$ in the counting space, the numbers of occurrence of all the directed reactions form a directed reaction cycle $\tilde{c}_{d}$ and we can thus define a map $\phi_{d}: \mathcal{C}_{\infty} \rightarrow \mathbb{R}^{2 M}$ with $\phi_{d}(c)$ being the directed reaction cycle generated in this way.

Combining the maps $\phi$ and $\phi_{d}$ we can actually have the commutative diagram below, with $\psi: \mathbb{R}^{2 M} \rightarrow \mathbb{R}^{M}$ mapping $\tilde{c}_{d}$ to $\tilde{c}$ in the way $\psi\left(c_{1}^{+}, c_{2}^{+}, \ldots, c_{M}^{+}, c_{1}^{-}, c_{2}^{-}, \ldots, c_{M}^{-}\right)=$ $\left(c_{1}^{+}-c_{1}^{-}, c_{2}^{+}-c_{2}^{-}, \ldots, c_{M}^{+}-c_{M}^{-}\right)$, followed by

$$
\omega_{\tilde{c}}=\sum_{\psi\left(\tilde{c}_{d}\right)=\tilde{c}} \omega_{\tilde{c}_{d}},
$$
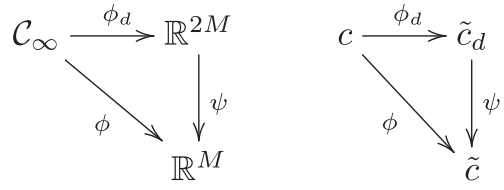

in which the directed reaction cycle flux of $\tilde{c}_{d}$ is

$$
\omega_{\tilde{c}_{d}}=\sum_{\phi_{d}(c)=\tilde{c}_{d}} \omega_{c} .
$$

Combined with cycle decomposition (B1) in the counting space, we can add up $\pi_{y} q_{y \tilde{y}}$ w.r.t. all possible states $y$ and obtain

$$
\sum_{y, \tilde{y} \in S, \tilde{y}=y+\Xi_{d, \ell_{d}}^{Y}} \pi_{y} q_{y \tilde{y}}=\sum_{\tilde{c}_{d} \in \phi_{d}\left(\mathcal{C}_{\infty}\right)} \omega_{\tilde{c}_{d}} \tilde{c}_{d}\left(\ell_{d}\right)
$$

for each $\ell_{d} \in\{1,2, \ldots, 2 M\}$, where $\Xi_{d, \ell_{d}}^{Y}$ is the $\ell_{d}$ th column of the matrix $\Xi_{d}^{Y}, \phi_{d}\left(\mathcal{C}_{\infty}\right)$ is the image of $\phi_{d}$ and $\tilde{c}_{d}\left(\ell_{d}\right)$ is the $\ell_{d}$ th entry of $\tilde{c}_{d}$.

Furthermore,

$$
\begin{aligned}
\sum_{y, \tilde{y} \in S, \tilde{y}=y+\Xi_{\ell}^{Y}}\left[\pi_{y} q_{y \tilde{y}}-\pi_{\tilde{y}} q_{\tilde{y} y}\right] & =\sum_{\tilde{c}_{d} \in \phi_{d}\left(\mathcal{C}_{\infty}\right)} \omega_{\tilde{c}_{d}} \psi\left(\tilde{c}_{d}\right)(\ell) \\
& =\sum_{\tilde{c} \in \phi\left(\mathcal{C}_{\infty}\right)} \omega_{\tilde{c}} \tilde{c}(\ell)
\end{aligned}
$$

where $\Xi_{\ell}^{Y}$ is the $\ell$ th column of the matrix $\Xi^{Y}, \phi\left(\mathcal{C}_{\infty}\right)$ is the image of $\phi$ and $\tilde{c}(\ell)$ is the $\ell$ th entry of $\tilde{c}$ with $\ell \in\{1,2, \ldots, M\}$.

\section{APPENDIX E: CYCLE DECOMPOSITION OF THE MACROSCOPIC FLUXES}

The left-hand side of Eqs. (D2) and (D3) are the mesoscopic averaged one-step flux and the net flux. So when we divide it by $V$ and let $V \rightarrow \infty$ in Eq. (D2), we can actually get the cycle decomposition for the macroscopic flux of the $\ell$ th reaction:

$$
J^{+\ell}=\sum_{\tilde{c}_{d} \in \phi_{d}\left(\mathcal{C}_{\infty}\right)} \mathcal{J}_{\tilde{c}_{d}} c_{\ell}^{+}
$$

and

$$
J^{-\ell}=\sum_{\tilde{c}_{d} \in \phi_{d}\left(\mathcal{C}_{\infty}\right)} \mathcal{J}_{\tilde{c}_{d}} c_{\ell}^{-},
$$

where $J^{+\ell}=\lim _{V \rightarrow \infty} \frac{\sum_{y, \tilde{y} \in S, \tilde{y}=y+\Xi_{d, \ell}^{Y}} \pi_{y} q_{y \tilde{y}}}{V}=\lim _{V \rightarrow \infty} \frac{\left\langle q_{y \tilde{y}}\right\rangle}{V}$ is the forward flux of the $\ell$ th reaction in the deterministic model and $\left\langle q_{y \tilde{y}}\right\rangle$ is the averaged $q_{y \tilde{y}}$ over $y$ with all $\tilde{y}=y+\Xi_{d, \ell}^{Y}$ [24,28]. $J^{-\ell}$ is the backward flux of the $\ell$ th reaction. $\mathcal{J}_{\tilde{c}_{d}}=$ $\lim _{V \rightarrow \infty} \frac{\omega_{\tilde{c}_{d}}}{V}$ is the macroscopic cycle currents for the directed reaction cycle $\tilde{c}_{d}$, where every $\tilde{c}_{d}$ is a $2 M$-dimensional vector with $\tilde{c}_{d}=\left(c_{1}^{+}, c_{2}^{+}, \ldots, c_{M}^{+}, c_{1}^{-}, c_{2}^{-}, \ldots, c_{M}^{-}\right)$.

When we divide both sides by $V$ and let $V \rightarrow \infty$ in Eq. (D3), we can get Eq. (7), i.e., the decomposition for the net macroscopic flux

$$
J^{\ell}=\sum_{\tilde{c} \in \phi\left(\mathcal{C}_{\infty}\right)} \mathcal{J}_{\tilde{c}} c_{\ell}
$$

where $J^{\ell}=\lim _{V \rightarrow \infty} \frac{\sum_{y, \tilde{y} \in S \tilde{y}=y+z_{\ell}^{Y}}\left[\pi_{y} q_{y \tilde{y}}-\pi_{\tilde{y}} q_{\tilde{y} y}\right]}{V}=J^{+\ell}-J^{-\ell}$ is the net macroscopic flux of the $\ell$ th reaction and $\mathcal{J}_{\tilde{c}}$ is the macroscopic cycle currents for the original reaction cycle $\tilde{c}$.

\section{APPENDIX F: RATIO $\frac{\omega_{\tilde{c}}}{\omega_{\tilde{c}_{-}}}$ONLY DEPENDS ON THE OVERALL EFFECT FOR GENERAL CRNS WITH MULTIPLE INPUT AND OUTPUT COMPLEXES}

We can further show below that the ratio $\frac{\omega_{\tilde{c}}}{\omega_{\tilde{c}_{-}}}$only depends on the overall effect, i.e., $\vec{l}=\left[\Xi_{b}^{X}, 0\right] \times \tilde{c}^{T}$.

Consider its $\operatorname{logarithm} F(\tilde{c})=\log \frac{\omega_{\tilde{c}}}{\omega_{\tilde{c}_{-}}}$, then $F$ is still linear with respect to $\tilde{c}$, with the similar argument in previous sections. So we can denote $F(\tilde{c})=\vec{v}^{T} \tilde{c}^{T}$.

Due to the Wegscheider's condition, we know that for any closed cycle, $F(\tilde{c})$ should be zero. To be precise for any $\tilde{c}$ satisfying,

$$
\left[\begin{array}{cc}
\Xi_{b}^{X} & 0 \\
\Xi_{b}^{Y} & \Xi_{i}^{Y}
\end{array}\right] \cdot \tilde{c}^{T}=0 \Rightarrow F(\tilde{c})=0 .
$$

Therefore, $\vec{v}$ is in the space spanned by the row vectors of the above matrix $\Xi$, i.e., there exists a vector $\vec{a}$ satisfying

$$
\vec{v}^{T}=\vec{a}^{T} \cdot\left[\begin{array}{cc}
\Xi_{b}^{X} & 0 \\
\Xi_{b}^{Y} & \Xi_{i}^{Y}
\end{array}\right] .
$$

Thus $F(\tilde{c})=\vec{v}^{T} \tilde{c}^{T}=\vec{a}^{T}\left[\begin{array}{l}\vec{l} \\ 0\end{array}\right]=\vec{a}_{X} \cdot \vec{l}$ where $\vec{a}_{X}$ consists of the former $N_{1}$ elements in $a$. 
[1] H. Qian, Chemical reaction kinetic perspective with mesoscopic nonequilibrium thermodynamics, in Complexity Science: An Introduction, edited by M. Peletier, R. van Santen, and E. Steur (World Scientific, Singapore, 2019), pp. 347-373.

[2] T. L. Hill, Studies in irreversible thermodynamics, VI, Proc. Nat. Acad. Sci. USA 56, 45 (1966).

[3] T. L. Hill, Free Energy Transduction and Biochemical Cycle Kinetics (Courier Corporation, North Chelmsford, MA, 2005).

[4] T. Hill and Y. Chen, Stochastics of cycle completions (fluxes) in biochemical kinetic diagrams, Proc. Nat. Acad. Sci. USA 72, 1291 (1975).

[5] G. E. Briggs and J. B. S. Haldane, A note on the kinetics of enzyme action, Biochem J. 19, 338 (1925).

[6] G. N. Lewis, A new principle of equilibrium, Proc. Nat. Acad. Sci. USA. 11, 179 (1925).

[7] G. E. Crooks, Entropy production fluctuation theorem and the nonequilibrium work relation for free energy differences, Phys. Rev. E. 60, 2721 (1999).

[8] G. Gallavotti and E. G. D. Cohen, Dynamical Ensembles in Nonequilibrium Statistical Mechanics, Phys. Rev. Lett. 74, 2694 (1995).

[9] H. Qian, Nonequilibrium steady-state circulation and heat dissipation functional, Phys. Rev. E 64, 022101 (2001).

[10] H. Qian and X. S. Xie, Generalized haldane equation and fluctuation theorem in the steady-state cycle kinetics of single enzymes, Phys. Rev. E 74, 010902 (2006).

[11] D. A. Beard and H. Qian, Relationship between thermodynamic driving force and one-way fluxes in reversible processes, Plos One 2, e144 (2007).

[12] J. Ross, Thermodynamics and Fluctuations far from Equilibrium (Springer, New York, 2008).

[13] R. Rao and M. Esposito, Nonequilibrium Thermodynamics of Chemical Reaction Networks: Wisdom from Stochastic Thermodynamics, Phys. Rev. X 6, 041064 (2016).

[14] D. A. McQuarrie, Stochastic approach to chemical kinetics, J. Appl. Prob. 4, 413 (1967).
[15] C. Qian, M. Qian, and M. P. Qian, Markov chain as a model of Hill's theory on circulation, Sci. Sin. 24, 1431 (1981).

[16] D. Q. Jiang, M. Qian, and M. P. Qian, Circulation Distribution, Entropy Production and Irreversibility of Finite Markov Chains with Continuous Parameter (Springer, Berlin, 2004), pp. 45-66.

[17] M. Feinberg, Lectures on chemical reaction networks, Ohio State University, School of Engineering, https://crnt.osu.edu/ LecturesOnReactionNetworks (1979).

[18] S. de Groot and P. Mazur, Non-Equilibrium Thermodynamics (Courier Corporation, North Chelmsford, MA, 2013).

[19] M. Peka, Thermodynamics and foundations of mass-action kinetics, Prog. Reaction Kinetics Mechanism 30, 3 (2005).

[20] D. Q. Jiang, M. Qian, and M. P. Qian, 1 Circulation Distribution, Entropy Production and Irreversibility of Denumerable Markov Chains (Springer, Berlin, 2004), pp. 11-44.

[21] S. L. Kalpazidou, Cycle Representations of Markov Processes (Springer, New York, 2006).

[22] M. Polettini and M. Esposito, Irreversible thermodynamics of open chemical networks. I. Emergent cycles and broken conservation laws, J. Chem. Phys. B 141, 024117 (2014).

[23] S. Schuster and R. Schuster, A generalization of wegscheider's condition. implications for properties of steady states and for quasi-steady-state approximation, J. Math. Chem. 3, 25 (1989).

[24] H. Ge and H. Qian, Mathematical formalism of nonequilibrium thermodynamics for nonlinear chemical reaction systems with general rate law, J. Stat. Phys. 166, 190 (2017).

[25] H. P. J. Bonarius, G. Schmid, and J. Tramper, Flux analysis of underdetermined metabolic networks: The quest for the missing constraints, Tibtech 15, 308 (1997).

[26] I. T. J. D. Orth and B. Palsson, What is flux balance analysis? Nat. Biotechnol. 28, 245 (2010).

[27] D. A. Beard, S. Liang, and H. Qian, Energy balance for analysis of complex metabolic networks, Biophys. J. 83, 79 (2002).

[28] T. G. Kurtz et al., Strong approximation theorems for density dependent markov chains, Stoch. Proc. Appl. 6, 223 (1978). 